REGARDS

SUR L'ECONOMIE ALLEMANDE

BULLETIN ECONOMIQUE DU CIRAC

\section{Regards sur l'économie allemande}

Bulletin économique du CIRAC

$91 \mid 2009$

Varia

\title{
RFA : repères chronologiques - politiques, économiques et sociaux
}

Isabelle Bourgeois et René Lasserre

\section{(2) OpenEdition}

Édition électronique

URL : http://journals.openedition.org/rea/3711

DOI : 10.4000/rea.3711

ISBN : 978-2-8218-0878-2

ISSN : 1965-0787

\section{Éditeur}

CIRAC

Édition imprimée

Date de publication : 1 mai 2009

Pagination : 57-82

ISSN : 1156-8992

Référence électronique

Isabelle Bourgeois et René Lasserre, «RFA : repères chronologiques - politiques, économiques et sociaux », Regards sur l'économie allemande [En ligne], 91 | mai 2009, mis en ligne le 01 mai 2011, consulté le 10 décembre 2020. URL : http://journals.openedition.org/rea/3711 ; DOI : https://doi.org/ 10.4000/rea.3711 


\title{
RFA : repères chronologiques - politiques, économiques et sociaux
}

\author{
Isabelle Bourgeois, René Lasserre
}

\section{Reconstruction et renouveau}

1948

20 juin : Dans les 3 zones d'occupation occidentales, réforme monétaire. Création du Deutsche Mark (DM) et institution de la Bank deutscher Länder, préfiguration de la Bundesbank qui sera créée le 01-08-1957. 21 juin : A l'initiative de Ludwig Erhard, Directeur de l'économie de la bizone, promulgation de la libéralisation des prix (Leitsätzegesetz). 23 juin : Dans la zone d'occupation soviétique, introduction du Mark est. 24 juin : Début du blocus de Berlin (il prend fin le 12 mai 1949).

10-23 août : Travaux de la Convention Constitutionnelle (Verfassungskonvent) à Herrenchiemsee qui rédige un projet de Constitution pour la partie ouest de l'Allemagne (3 zones d'occupation). $1^{\text {er }}$ septembre : Constitution à Bonn du Conseil Parlementaire (Parlamentarischer Rat).

\section{9}

8 mai : Adoption de la Loi fondamentale (Grundgesetz), Constitution de la future RFA. 23 mai : Proclamation de la Loi fondamentale. 24 mai : Création de la République fédérale d'Allemagne (RFA). 14 août : Elections au Bundestag qui élit Konrad Adenauer (CDU) chancelier le 15-09; réélu plusieurs fois, il assume ces fonctions jusqu'en 1963. 12 septembre : Theodor Heuss (FDP) est élu Président de la RFA.

7 octobre : Constitution de la République démocratique allemande (RDA) à l'est. Lors du $3^{\mathrm{e}}$ Congrès du parti SED en juillet 1950 qui adopte la doctrine marxisteléniniste, le Comité central nouvellement constitué élit son secrétaire général Walter Ulbricht.

28 janvier : A l'ouest, constitution de la confédération patronale Bundesvereinigung der Deutschen Arbeitgeberverbände (BDA). 9 avril : Adoption de la Loi sur les conventions collectives (Tarifvertragsgesetz) qui ne s'applique tout d'abord que dans les zones britannique et américaine. La loi ne sera généralisée pour l'ensemble de la République fédérale que le 23 avril 1953. 12-14 octobre : Constitution de la Confédération des syndicats allemands Deutscher Gewerkschaftsbund (DGB). 19 octobre : Constitution de la fédération allemande de l'industrie Bundesverband der Deutschen Industrie (BDI).

27 octobre : Constitution de la fédération des chambres de commerce et d'industrie Deutscher Industrie- und Handelskammertag (DIHT).

1950

22 mars : Fort du soutien des Etats-Unis, le chancelier Adenauer réclame publiquement l'organisation d'élections en RFA et en RDA en vue de la réunification de l'Allemagne.

25 juin : Début de la guerre de Corée. En réaction au risque d'une " $3^{\mathrm{e}}$ Guerre mondiale », le Conseil de l'Europe décide le 18 août de constituer une " armée de l'Europe » intégrant des contingents allemands. 8 juillet : La RFA devient

Constitution de la RFA et de la RDA

Renaissance des partenaires sociaux 
Statut de Berlin

Création du Tribunal constitutionnel fédéral

Première loi sur la cogestion

Rétablissement de l'assurance chômage

Refondation de la démocratie d'entreprise

Insurrection en RDA membre associé du Conseil de l'Europe ; elle en deviendra membre plein et entier le 2 mai 1951. La contribution de la RFA à la défense européenne incite le chancelier Adenauer à plaider pour une révision du statut d'occupation et un recouvrement progressif de la souveraineté de la RFA.

28 septembre : La RDA intègre le COMECON.

1er octobre : Berlin est désormais un Land de la RFA (statut de Berlin). Les lois de la RFA s'y appliquent, à l'exception des dispositions contraires au Statut quadripartite (par exemple le service militaire).

20 décembre : Adoption de la Loi d'assistance aux victimes de guerre (Kriegsopferversorgung).

\section{1}

12 mars : Adoption de la loi fédérale portant création du Tribunal constitutionnel fédéral (Bundesverfassungsgericht). Il sera institué à Karlsruhe le 28-09.

18 avril : Signature à Paris du Traité sur la CECA.

21 mai : Adoption, sous la pression du DGB, de la première loi sur la cogestion dans le secteur charbon-acier (Montanmitbestimmungsgesetz) qui perpétue le dispositif mis en place par les Alliés en mars 1947.

2 août : Institution de la Deutsche Forschungsgemeinschaft (DFG), organisation centrale de soutien à la recherche, au statut de collectivité de droit public et dotée du pouvoir d'auto-administration.

10 août : La RFA adhère au traité du GATT.

\section{2}

10 mars : Institution à Nuremberg de l'Office fédéral de placement et d'assurance chômage (Bundesanstalt für Arbeitsvermittlung und Arbeitslosenversicherung). Cette collectivité de droit public autonome est co-gérée par les partenaires sociaux qui ont vu leurs droits d'auto-administration dans les organismes d'assurance sociale rétablis par la loi le 22-02-1951.

26 mai : Entrée en vigueur prévue du Traité sur l'Allemagne (Deutschlandvertrag) conclu entre les 3 puissances alliées et le chancelier Adenauer. Mais ce traité étant conditionné à l'entrée en vigueur de la Communauté de défense européenne, que la France a rejetée en août 1954, le Deutschlandvertrag est révisé à Paris en octobre 1954. Les nouvelles dispositions (Accords de Paris) prévoient la fin du régime d'occupation de la RFA et son accueil au sein de l'Union de l'Europe occidentale (UEO) et de l'OTAN.

23 juillet : Centralisation administrative de la RDA (Loi de démocratisation abolissant les Länder) et collectivisation.

14 août : RFA : Loi sur la péréquation des charges (Lastenausgleich) permettant de contribuer à l'indemnisation des dommages et pertes subis du fait des destructions et des expulsions liées à la guerre. La péréquation est fondée sur une contribution annuelle de solidarité imposée à tous les détenteurs de patrimoine épargnés par la guerre et étalée sur une période de 27 ans.

11 octobre : Adoption en RFA de la Loi sur la Constitution interne de l'entreprise (Betriebsverfassungsgsetz) attribuant aux Conseils d'entreprise des droits de codécision très étendus et instaurant une représentation au tiers des sièges dans les conseils de surveillance des entreprises de plus de 500 salariés.

25 décembre : Lancement de la télévision en RFA : naissance de la première chaîne, réalisée par le Groupement de travail des établissements de radiodiffusion de droit public des Länder (ARD) créé par contrat en 1950.

\section{3}

17 juin : Insurrection en RDA contre la hausse des normes de production, répression sanglante par l'armée soviétique. La RFA choisit cette date comme fête nationale. 
1954

4 juillet : La RFA remporte la Coupe du Monde de football (« miracle de Berne »).

1955

5 mai : La RFA recouvre en large partie sa souveraineté en application des Accords de Paris. 14 mai : En réaction à l'entrée de la RFA dans l'OTAN, la RDA devient membre du Pacte de Varsovie.

5 août : Adoption de la Loi fédérale de représentation du personnel (Bundespersonalvertretungsgesetz) qui accorde aux fonctionnaires et employés de la fonction publique des droits de représentation comparables à ceux des salariés du privé, garantis par le Betriebsverfassungsgesetz de 1952, mais à portée essentiellement consultative.

8-14 septembre : Voyage d'Adenauer à Moscou. " Normalisation » et établissement de relations diplomatiques entre la RFA et l'URSS. Retour des prisonniers allemands.

\section{6}

17 août : Arrêt du Tribunal constitutionnel fédéral (TCF) interdisant le parti communiste (Kommunistische Partei Deutschlands, DKP) en RFA. En 1952, la Cour avait interdit le parti néo-nazi Sozialistische Reichspartei (SRP).

$1^{\text {er }}$ mai : Le DGB lance sa campagne pour la semaine de 5 jours et l'objectif des 40 heures hebdomadaires. Le 25 juillet, le syndicat IG Metall signe à Brême un accord cadre qui prévoit la réduction de 48 à 45 heures dans l'industrie des métaux.

\section{7}

$1^{\mathrm{er}}$ janvier : La Sarre, après referendum, réintègre la RFA.

16 janvier : Le TCF (« jugement Elfes ») considère le " droit au libre épanouissement de la personnalité » (art. $2, \S 1$ de la Loi fondamentale) comme le premier de tous les droits fondamentaux (explicitement ancrés dans la Constitution ou non), organisant ainsi ceux-ci en un système de valeurs cohérent.

Février : Parution de l'ouvrage de Ludwig Erhard: “Wohlstand für Alle » (« La prospérité pour tous ») qui popularise la doctrine ordo-libérale de l'économie de marché sociale (soziale Marktwirtschaft).

23 février : Réforme du régime de l'assurance-retraite dont l'organisation remontait à la Reichsversicherungsordnung de 1911, instaurant un mode de financement fondé sur une assiette de cotisation plus large des assurés ; elle permet une revalorisation substantielle des retraites et l'indexation de ces dernières sur l'évolution générale des revenus salariaux. La réforme inaugure la refondation de l'Etat social initié dans les années 1880 par Bismarck et le développement continu que connaîtra le système de protection sociale jusque dans les années 1980. Cette première avancée sera suivie le $\mathbf{2 6}$ juin par l'adoption de la loi garantissant le maintien du salaire des ouvriers en cas d'arrêt maladie pendant six semaines (Lohnfortzahlung) dont le principe fut obtenu à la suite de la grève menée dans l'industrie des métaux du Schleswig-Holstein par IG Metall d'octobre 1956 à février 1957.

25 mars : Signature des Traités de Rome instituant la Communauté économique européenne (CEE ; entrée en vigueur le 01-01-1958) et la Communauté européenne de l'énergie atomique (Euratom).

27 juillet : Adoption de la Loi contre les entraves à la concurrence (Gesetz gegen Wettbewerbsbeschränkungen), entrée en vigueur le 01-01-1958 et considérée depuis comme la 'Loi fondamentale' de l'économie sociale de marché ; institution de l'Office fédéral des Cartels (Bundeskartellamt) le même jour.

$1^{\text {er }}$ août : Entrée en vigueur de la Loi sur la Bundesbank (Gesetz über die Bundesbank) du 26-07 portant création de la nouvelle banque centrale de la RFA dotée d'un statut qui garantit son indépendance par rapport au gouvernement fédéral.
« Miracle de Berne»

La RFA recouvre une grande partie de sa souveraineté

Interdiction du parti communiste en RFA

Début de la réduction de la durée du travail

Les droits fondamentaux, un système de valeurs cohérent

Réorganisation du système d'assurance retraite

Loi contre les entraves à la concurrence

Création de la Bundesbank 
Egalité des sexes

Le SPD abandonne la référence au marxisme

Création de Deutsche Welle et Deutschlandfunk

Constitutionnalisation de l'indépendance des médias

$1^{\text {ere }}$ réévaluation du DM

Construction du Mur de Berlin

Création de l'aide sociale

\section{8}

15 janvier : «Arrêt Lüth » (Lüth-Urteil) du Tribunal constitutionnel fédéral, qui érige les libertés fondamentales garanties par la Constitution au rang de principes objectifs supérieurs régissant le droit, conférant ainsi à la Loi fondamentale valeur « d'ordre objectif des valeurs » (objektive Wertordnung).

$1^{\mathrm{er}}$ juillet : L'égalité des sexes est inscrite au Code civil (Bürgerliches Gesetzbuch, $B G B$ ) à la suite d'un arrêt rendu le 19-12-1953 par le TCF qui avait donné vie à ce droit fondamental inscrit à l'art. 3 , § 2 de la Loi fondamentale.

\section{9}

24 mars : Les premières actions populaires (Volksaktien) sont émises lors de l'ouverture du capital de Preussag qui inaugure la privatisation partielle d'une série de sociétés appartenant à l'Etat fédéral (Volkswagen en juillet 1960, Veba en avril 1965). L'objectif de cette politique est d'ouvrir l'accès à l'actionnariat populaire et de favoriser l'épargne, grâce à l'adoption, le 5 mai, de la Loi sur la prime à l'épargne (Sparprämiengesetz). Le « miracle économique » place l'attention sur la constitution de patrimoine aux mains des salariés après que la réforme monétaire de 1948 avait bénéficié en priorité au capital productif.

13-15 novembre : Congrès de Bad Godesberg au cours duquel le parti socialdémocrate (SPD) abandonne la référence exclusive au marxisme et déclare se réclamer également de l'humanisme chrétien et des valeurs de l'économie sociale de marché (liberté, propriété, égalité des droits, solidarité...). Dans son programme fondamental adopté alors, le SPD décrit ainsi son cheminement: « Le parti social-démocrate, issu d'un parti de la classe ouvrière, est devenu un parti populaire ».

\section{0}

26 octobre : Adoption de la première Loi de promotion de la constitution d'un patrimoine (Gesetz zur Förderung der Vermögensbildung); elle entre en vigueur le 12-07-1961.

29 novembre : Création, par une loi fédérale, de la radio internationale Deutsche Welle et de la radio Deutschlandfunk dont les émissions sont destinées à l'Europe (et à la RDA). Après l'Unité, cette dernière deviendra une radio 'nationale'.

\section{1}

28 février : « $1^{\text {er }}$ Jugement sur la radiodiffusion » rendu par le TCF (surnommé « Arrêt sur la TV Adenauer »). II définit le droit constitutionnel fondamental de la liberté de la radiodiffusion, précise l'indépendance de tous les médias par rapport à l'Etat (exécutif) et renforce la compétence législative des Länder en matière d'audiovisuel. 6 juin : Création, par Contrat d'Etat conclu entre tous les Länder, de leur établissement de radiodiffusion de droit public commun : Zweites Deutsches Fernsehen (ZDF), qui lancera la $2^{\mathrm{e}}$ chaîne de TV le $1^{\mathrm{er}}$ avril 1963.

6 mars : Réévaluation du Deutsche Mark de 4,75 \% : par cette première correction de parité depuis 1948, la Deutsche Bundesbank remédie à la sous-évaluation devenue manifeste du DM qui, en générant des excédents importants du commerce extérieur et un afflux croissant de devises, contribuait à une inflation importée préjudiciable à la stabilité des prix.

13 août : Début de la construction du Mur de Berlin.

\section{2}

$1^{\mathrm{er}}$ juillet : Entrée en vigueur de la Loi fédérale sur l'aide sociale (Bundessozialhilfegesetz) qui garantit à tout citoyen un revenu minimum d'assistance en cas de détresse sociale.

\section{3}

22 janvier : Signature du Traité de l'Elysée entre la République française et la RFA. 
Avril-mai : Grève dans l'industrie des métaux du Bade-Wurtemberg, par laquelle IG Metall relance l'offensive en vue de la semaine de 40 heures. Après 2 semaines de conflit (avec lock-out patronal), IG Metall obtient une réduction de $42 \mathrm{~h} 30$ à $41 \mathrm{~h} 15$ au $1^{\mathrm{er}}$ janvier 1964. Du fait de la récession de 1966, les 40 heures hebdomadaires ne seront obtenues dans la branche-pilote des métaux qu'au 01-01-1967.

14 août : Adoption de la Loi portant création du Conseil des experts pour l'examen de l'évolution économique globale (Sachverständigenrat zur Begutachtung der gesamtwirtschaftlichen Entwicklung), composé d'experts scientifiques issus de la société civile et mieux connu sous le nom de Conseil des Sages. Sa mission institutionnelle d'évaluation d'intérêt public lui confère le statut d'une haute autorité.

16 octobre : Ludwig Erhard (CDU) succède à Konrad Adenauer (CDU) comme chancelier.

\section{5}

6 septembre: Adoption de la Loi des sociétés par actions (Aktiengesetz), qui modernise le texte initial de 1937 et entre en vigueur le 01-01-1966.

\section{Transition réformatrice et alternance}

\section{6}

5 août : Jugement du TCF ( « Arrêt Der Spiegel ») qui étend le droit fondamental de la liberté de l'information (art. 5 de la Loi fondamentale) à la presse en tant qu'institution et constitutionnalise ainsi la liberté de la presse.

27 octobre : Le FDP quitte la coalition gouvernementale pour raison de désaccord avec la politique économique de la CDU/CSU qui, dans le projet de budget pour 1967, préconise de poursuivre l'objectif de l'équilibre budgétaire en dépit de la récession conjoncturelle qui s'est amorcée au cours de l'été. 30 novembre : A la suite de la démission du chancelier L. Erhard est formé un gouvernement de Grande Coalition (CDU/SPD) dans lequel le SPD accède aux responsabilités gouvernementales. Kurt Georg Kiesinger (CDU) est élu chancelier, Willy Brandt (SPD) devient vice-chancelier et ministre des Affaires étrangères de la RFA, tandis que Karl Schiller (SPD) devient ministre fédéral de l'Economie et F.-J. Strauß (CSU) ministre des Finances.

\section{7}

14 juin : Entrée en vigueur de la Loi pour la promotion de la stabilité et de la croissance (Gesetz zur Förderung der Stabilität und des Wachstums der Wirtschaft). Elle stipule qu'il revient à l'Etat d'intervenir au plan économique pour promouvoir une croissance durable et équilibrée et, à cette fin, de concilier quatre objectifs: stabilité des prix, taux d'emploi élevé, croissance, équilibre des échanges extérieurs. En appelant à un rôle plus actif de l'Etat dans le « pilotage global » de l'économie, cette loi marque un tournant keynésien au plan doctrinal par rapport à l'orthodoxie ordo-libérale en vigueur depuis 1948. Elle reste néanmoins compatible avec l'approche du libéralisme organisé en ce sens que l'Etat se contente de promouvoir un équilibre macro-économique et des orientations d'ensemble, tandis que l'activité économique continue de relever de l'initiative des acteurs privés et des lois du marché. La loi renforce également les missions du Conseil des Sages, chargé depuis "d'évaluer l'évolution économique [du pays] et d'éclairer le jugement de toutes les instances responsables en matière de politique économique ainsi que celui de l'opinion publique ". Dans ce cadre, l'Etat fédéral se voit confier la responsabilité de promouvoir une politique économique incitative fondée sur la concertation et le dialogue entre les acteurs économiques publics et privés.

24 juillet : Loi sur les Partis politiques (Parteiengesetz) qui en définit le statut, les missions, les principes de fonctionnement démocratique interne, les règles de gestion et de financement.
Avancée vers la semaine de 40 heures

Création du Conseil des Sages

Ludwig Erhard chancelier

Loi sur les sociétés par actions

Constitutionnalisation de la liberté de la presse

Gouvernement de Grande Coalition

Loi pour la promotion de la stabilité et de la croissance 
1968

nouvelle Constitution

RFA : manifestations étudiantes

Réforme de la Constitution financière fédérale

Réorganisation de l'assurance/assistance chômage

Homogénéisation du système de formation professionnelle

Willy Brandt chancelie

Début de l'ouverture à l'Est

Création d'une allocation d'études

Willy Brandt Prix Nobel de la Paix $\mathbf{1}^{\mathrm{er}}$ janvier : Création de la TVA en RFA.

6 avril : Adoption en RDA par referendum de la Constitution de 1968 qui instaure les bases d'un système socialiste et institue la RDA comme «Etat socialiste de nation allemande $»$.

Pâques 1968 : Manifestations étudiantes à Berlin-Ouest et attentat contre Rudi Dutschke, leader de la contestation étudiante, mouvement de protestation de grande ampleur dans les grandes villes universitaires de RFA. $\mathbf{3 0}$ mai : adoption par le Bundestag des Lois d'exception à mettre en œuvre en cas d'état d'urgence (Notstandsgesetze).

$\mathbf{1}^{\mathrm{er}}$ juillet : Entrée en vigueur de l'Union douanière au sein de la CEE.

\section{9}

5 mai : Election de Gustav Heinemann, ministre de la Justice (SPD) à la présidence de la République, avec le soutien du FDP contre le ministre de la Défense Gerhard Schröder, candidat de la CDU.

12 mai : Réforme de la Constitution financière fédérale (Finanzverfassung), initiée par le ministre fédéral des Finances, Franz-Josef Strauß, et instituant une nouvelle répartition des recettes fiscales entre Bund, Länder et Communes.

$1^{\text {er }}$ juillet : Promulgation de la loi sur la promotion du travail (Arbeitsförderungsgesetz, AFG) qui modernise le placement et l'indemnisation des chômeurs (restaurés en 1952) et met en place un système de guichet unique qui regroupe, sous l'égide de la Bundesanstalt für Arbeit et de ses offices régionaux et locaux du travail (Arbeitsämter), le placement, les mesures de formation-reclassement et un système rénové d'assurance et d'assistance chômage.

14 août : Loi sur la formation professionnelle (Berufsbildungsgesetz) qui met en place un système fédéral homogène de formation professionnelle initiale en alternance, prévoyant des règlements d'apprentissage concertés entre l'Etat fédéral, les Länder et les partenaires sociaux, des contrats d'apprentissage sous la responsabilité des entreprises garantissant une rémunération et les droits sociaux des apprentis.

21 octobre : Willy Brandt (SPD) est élu chancelier à la tête d'un gouvernement de coalition SPD/FDP à la suite des élections au Bundestag du 28 septembre, où les deux partis obtiennent ensemble une courte majorité. 28 octobre : Dans sa déclaration gouvernementale, Willy Brandt annonce un vaste programme de réformes intérieures et de mesures sociales et les grandes lignes de sa politique à l'Est.

24 octobre : Révaluation du DM de 8,5\%.

\section{0}

$1^{\text {er }}$ janvier : Amélioration et généralisation pour tous les salariés des conditions de maintien du salaire en cas de maladie (Lohnfortzahlung).

12 août : Signature à Moscou du Traité germano-russe.

7 décembre : Signature à Varsovie du Traité germano-polonais (le chancelier Brandt honore la mémoire des victimes du ghetto de Varsovie).

\section{1}

26 août : Loi sur le soutien à la formation initiale et aux études (Bundesausbildungsförderungsgesetz, BAföG) qui instaure un droit individuel à la poursuite d'études par l'attribution d'allocations scolaires et d'enseignement supérieur.

10 décembre : Willy Brandt se voit décerner le Prix Nobel de la Paix pour son Ostpolitik.

17 décembre : $2^{\mathrm{e}}$ réévaluation du $\mathrm{DM}$, de $13,8 \%$ par rapport au $\$$, qui fait suite à la suspension de la convertibilité-or du \$ le 15 août 1971 par le président Nixon. 


\section{2}

15 janvier : Réforme de la Loi sur la Constitution interne de l'entreprise : les droits de co-décision des Conseils d'entreprise sont élargis, les droits des apprentis et jeunes salariés améliorés, la présence syndicale reconnue.

3 juin : Signature de l'accord quadripartite sur Berlin, ouvrant la voie à la normalisation des relations entre la RFA et la RDA.

17 juillet : Le Bundestag ayant refusé de voter le projet de budget fédéral (le 2804 ) et les débats budgétaires ayant été reportés sine die, démission du ministre fédéral des Finances Karl Schiller ; Helmut Schmidt le remplace.

21 septembre : Réforme du régime des retraites: instauration de la retraite flexible, extension du régime des retraites aux travailleurs indépendants et aux femmes inactives.

19 octobre : L'écrivain Heinrich Böll se voit décerner le Prix Nobel de littérature. C'est le premier Prix Nobel à un écrivain allemand depuis 1929 (Thomas Mann). Günter Grass sera le second (il l'obtiendra en 1999).

19 novembre : Elections anticipées au Bundestag à la suite de l'échec de la motion de défiance constructive (27 avril) initiée par l'opposition CDU/CSU contre la politique à l'Est. Fort de sa victoire électorale, Willy Brandt est confirmé dans ses fonctions de chancelier et la coalition SPD/FDP reconduite.

21 décembre : Signature du Traité fondamental (Grundlagenvertrag) entre la RFA et la RDA, pièce maîtresse de l'Ostpolitik. Dans son arrêt du 31-07-1973, le TCF déclare ce traité conforme à la Loi fondamentale, rappelant toutefois que toute reconnaissance pleine et entière en droit international de la RDA reste exclue. La Loi fondamentale (art. 23) continue de prévoir à terme l'Unité allemande.

\section{De Willy Brandt à Helmut Schmidt : entre réforme et gestion de crises}

\section{3}

La situation économique de surchauffe et les tensions inflationnistes entretenues par l'instabilité monétaire internationale conduisent le gouvernement à adopter deux plans de stabilisation successifs fondés sur des mesures fiscales restrictives en février et en mai.

11 mars : Réévaluation de $3 \%$ puis flottement du DM par rapport au Dollar (suivie d'une seconde réévaluation de 5,5\% en juin) avec maintien des parités fixes au sein du serpent monétaire européen institué en mars 1972.

Printemps: Grèves spontanées dans l'industrie pour l'obtention de primes de maintien du pouvoir d'achat. Les hausses salariales 1973 atteignent les $12 \%$.

Automne : Les pays de l'OPEC décident de quadrupler le prix du pétrole $\left(1^{\mathrm{er}}\right.$ choc pétrolier). Le gouvernement fédéral adopte un programme énergétique de substitution fondé sur la relance du charbon, le développement du nucléaire et les économies d'énergie. Mesures restrictives ponctuelles à la consommation de carburant et à la circulation.

\section{4}

$1^{\mathrm{er}}$ janvier : Amélioration du régime des prestations de l'assurance maladie pour différentes catégories de prestataires, intégration des handicapés et des étudiants parmi les bénéficiaires.

Février : Grève de trois jours dans les services publics. Les employés du secteur public obtiennent une augmentation salariale de $11 \%$ avec un forfait minimal de 170 DM qui est diversement appréciée par l'opinion.

16 mai : Démission du chancelier Brandt à la suite de l'affaire Guillaume. Helmut Schmidt, ministre fédéral de l'économie, (SPD) lui succède.
Helmut Schmidt, ministre fédéral des Finances

Réforme du régime des retraites

Traité fondamental entre la RFA et la RDA

$1^{\text {er }}$ choc pétrolier

Helmut Schmidt chancelier 
Lancement de la politique de soutien au Mittelstand

Début de la politique de soutien à l'économie et de lutte contre le chômage

Loi cadre pour l'enseignement supérieur

Généralisation et constitutionnalisation de la cogestion

Helmut Schmid reconduit chancelier
8 octobre : La Bavière adopte une Loi sur le soutien aux PME (Mittelstandsförderungsgesetz) qui servira de modèle aux autres Länder, puis au Bund dans le cadre du développement d'une politique fédérale de soutien aux PME où se conjuguent aides publiques (subventions d'investissement, prêts bonifiés, soutien à l'innovation, etc.) et une action plus globale visant à créer un environnement favorable à l'activité.

Décembre : Pour contenir la hausse du chômage liée à la crise pétrolière et aux mutations structurelles de l'industrie, le gouvernement Schmidt opère un changement de cap et lance un programme de soutien à la conjoncture, fondé sur l'investissement public et privé, le soutien à la demande des ménages par des allègements fiscaux et la revalorisation des prestations sociales. Cette politique de relance keynésienne financée par l'endettement a pour but principal de lutter contre la montée du chômage.

\section{5}

Août : Nouveau programme de soutien spécifique à l'investissement, principalement en faveur du BTP.

Le chômage franchit la barre du million de demandeurs d'emploi (4,6 \% des actifs).

15 Juin : Initiative conjointe de Helmut Schmidt et Valéry Giscard d'Estaing de création du G7 en vue de promouvoir une concertation économique mondiale.

\section{6}

$1^{\mathrm{er}}$ janvier : Entrée en vigueur des dispositions générales du Code social (Sozialgesetzbuch, $S G B$ ) codifiant l'ensemble des droits à prestations des assurés.

26 janvier : Adoption de la Loi cadre pour l'enseignement supérieur (Hochschulrahmengesetz, HRG) qui instaure, pour l'ensemble de la fédération, des principes communs pour l'organisation des études supérieures et des établissements d'enseignement supérieur. Conformément au principe de la souveraineté éducative et culturelle des Länder, il revient cependant à chacun de ces derniers de compléter ce cadre en se donnant une loi propre.

4 mai : Adoption de la Loi sur la cogestion paritaire (paritätische Mitbestimmung) instaurant une représentation des salariés à parité de droits et de sièges dans les conseils de surveillance des entreprises de plus de 2000 salariés. La loi, qui entre en vigueur au 01-01-1978, préserve néanmoins la prééminence du capital dans la prise de décision, puisque le président du Conseil de surveillance élu par les actionnaires dispose d'une voix décisive en cas de partage égal des voix. Un recours patronal est néanmoins déposé auprès du Tribunal constitutionnel pour atteinte à l'autonomie contractuelle et au droit de propriété.

Dans son « Jugement sur la cogestion » (Mitbestimmungsurteil) du 01-03-1979, le TCF déclare la loi conforme à la Loi fondamentale, puisqu'en donnant une voix prépondérante au capital en dernière instance, elle respecte le principe de la libre disposition du droit de propriété. Elle consacre en cela le principe de l'équilibre des forces entre capital et travail en instituant entre elles un mode de gestion coopératif que le TCF érige ainsi implicitement en principe constitutionnel.

Dans le même jugement, les juges clarifient également le rôle de la Cour suprême : elle n'a pas à connaître de political questions (contrairement à son homologue américaine). Elle n'intervient qu'en dernier recours pour le contrôle en conformité des lois avec la Constitution, s'interdisant d'interférer dans les choix politiques des législateurs en matière de politique économique, respectant ainsi la liberté d'agir (ou de se tromper) du législateur qui doit assumer ses responsabilités face à la société civile.

3 octobre : Elections fédérales pour le $8^{\mathrm{e}}$ Bundestag. La CDU progresse de près de $4 \%$ et, avec $48,6 \%$ des voix, redevient le premier parti. La coalition sociale-libérale garde néanmoins une majorité de 10 voix. Helmut Schmidt est reconduit comme chancelier le 15 décembre. 
1977

Mars : Nouveau « Programme de soutien à la croissance et à l'investissement d'avenir pour l'environnement » (Programm für wachstums-und umweltpolitische Zukunftsinvestition), principalement en faveur de la modernisation des transports et des économies d'énergie.

Septembre : «Paquet fiscal » prévoyant des allègements d'impôts en faveur des ménages et des entreprises ; ces mesures sont financées par l'endettement.

5 septembre : L'enlèvement puis l'assassinat de Hanns Martin Schleyer, président de la fédération du patronat (BDA) et de la fédération de l'industrie (BDI), par l'organisation terroriste Rote Armee Fraktion (RAF), marque le point culminant des années du terrorisme en RFA.

\section{8}

Juin : Mesures de soutien en faveur des mines et de la sidérurgie.

Juillet : $4^{e}$ sommet du $\mathrm{G} 7$ à Bonn par lequel les chefs d'Etat s'engagent à relancer l'économie mondiale par des mesures de soutien à hauteur d'environ $1 \%$ de leur PIB.

Juillet : En RFA, mesures de soutien à la demande des ménages : augmentation des allocations familiales et allègement de l'impôt sur le revenu.

5 décembre : A l'initiative conjointe de H. Schmidt et V. Giscard d'Estaing, le Conseil européen de Bruxelles décide la création du Système monétaire européen, lequel instaure dans l'espace de la CE une zone de changes fixes et entrera en vigueur le 13 mars 1979.

1979

12 décembre : Adoption, sous l'influence active de $\mathrm{H}$. Schmidt, de la double résolution de l'OTAN mettant en balance le renforcement futur du potentiel de fusées nucléaires américaines stationnées en Europe avec les progrès des négociations américano-soviétiques sur la réduction des euro-missiles. Cette résolution suscite une forte opposition au sein du SPD et de la gauche pacifiste.

\section{0}

5 octobre : Elections fédérales. Sous la direction de F.-J. Strauß comme candidat chancelier, la CDU/CSU perd $4,1 \%$ et retombe à $44,5 \%$ des voix, son plus mauvais score depuis 1949. Et tandis que le SPD progresse légèrement à $42,9 \%$, le FDP se hisse à 10,6 \%, ce qui permet à la coalition SPD/FDP de consolider sa majorité. Helmut Schmidt est réélu chancelier le 5 novembre et annonce un retour progressif à l'équilibre financier.

Mais alors que l'économie allemande avait commencé à se redresser et le chômage à diminuer en 1979 , le $2^{\mathrm{e}}$ choc pétrolier enclenché par la crise iranienne fait progressivement replonger le pays dans une récession sévère. La politique économique oscillera désormais entre un cap de stabilisation financière réclamé par les libéraux et un soutien à l'économie qu'exigent le recul de l'activité $(0 \%$ et $-1,1 \%$ en 1981/82) et la brusque remontée du chômage à 1,2 puis 1,8 million de demandeurs d'emploi.

\section{Du tournant libéral au réajustement structurel}

\section{1-1982}

10 octobre 1981 : Grande manifestation pacifiste à Bonn contre l'installation des fusées Pershing, à laquelle participent plusieurs responsables du SPD.

26 octobre 1981 : Après une série de mesures d'allègement fiscal pour les revenus moyens au $1^{\mathrm{er}}$ janvier, la coalition sociale-libérale s'accorde sur un programme de consolidation des finances de l'Etat et de la sécurité sociale qui prévoit la suppression d'exonérations fiscales et de subventions, ainsi que des économies bud-
Assassinat de Hanns Martin Schleyer

Relance concertée de l'économie

Création du

Système monétaire européen

Double résolution de I'OTAN

Helmut Schmidt

entame son $3^{\mathrm{e}}$ mandat

Deuxième choc pétrolier et nouvelle récession

Une politique économique contradictoire 
Rupture de la coalition sociale-libérale

Départ

d'Helmut Schmidt

Helmut Kohl confirmé chancelier dans les urnes

Mesures de consolidation budgétaire et sociale

Crise du SME et réévaluation du DM

Décision d'installation des Pershing gétaires et des réductions de prestations sociales qui entrent en vigueur au 01-011982.

3 février 1982 : Pour faire face à la détérioration préoccupante de l'emploi, le gouvernement adopte un compromis sur une « initiative commune pour les emplois, la croissance et la stabilité » qui, après avoir été approuvé par le Bundestag mais rejeté par le Bundesrat, est finalement adopté le 28 mai en commission de conciliation après abandon du projet de relèvement de la TVA.

Printemps 1982 : Le SPD perd plusieurs élections régionales.

Septembre 1982 : Les perspectives d'aggravation des déficits et de l'endettement dans le budget 1983 attisent la controverse au sein de la coalition gouvernementale. Le comte Otto Lambsdorff, ministre fédéral de l'Economie (FDP), remet au Chancelier un Mémorandum stratégique préconisant un changement de cap économique : en creusant la dette, la politique de soutien public à l'activité conduit à l'impuissance et doit faire place à une politique de réduction sévère des dépenses publiques et des prestations sociales. Cette divergence scelle la rupture de la coalition et ouvre la voie à une majorité nouvelle.

$1^{\text {er }}$ octobre : Le Bundestag vote une motion de défiance constructive et élit Helmut Kohl (CDU) comme successeur du chancelier Helmut Schmidt (SPD), ce qui met fin à une longue période de coalition sociale-libérale SPD/FDP (1969-1982). Le 4 octobre, $\mathrm{H}$. Kohl constitue son premier gouvernement de coalition CDU/CSUFDP et annonce une « politique de renouveau », fondée sur un recentrage du rôle de l'Etat et la responsabilité individuelle. Le 17 décembre, Helmut Kohl pose la question de confiance pour laquelle sa majorité s'abstient, ce qui ouvre la voie à la dissolution du Bundestag par le président Karl Carstens le 7 janvier 1983 et à des élections fédérales anticipées.

\section{3}

20 janvier : Discours de François Mitterrand devant le Bundestag à l'occasion du $20^{\mathrm{e}}$ anniversaire du Traité franco-allemand, au cours duquel il exprime son soutien à la position officielle allemande sur la question du stationnement des fusées Pershing.

6 mars : Elections fédérales anticipées qui confirment la nouvelle majorité CDU/CSU-FDP. Le SPD tombe à 38,2 \% des voix. Avec $5,6 \%$ des voix, les Verts font leur entrée au Bundestag. 29 mars : Helmut Kohl est réélu chancelier. II le restera jusqu'en 1998.

Mise en œuvre des nouvelles mesures de consolidation financière inscrites dans la loi budgétaire modificative pour 1983 . Au $1^{\mathrm{er}}$ janvier : hausse des cotisations à l'assurance chômage, diminution des prestations familiales et de l'aide au logement, réintroduction de la cotisation des retraités à l'assurance maladie, report de la revalorisation des retraites et de l'aide sociale. Au $1^{\text {er }}$ juillet : augmentation de la TVA de 13 à $14 \%$. Au $1^{\text {er }}$ août : entrée en vigueur de la réforme de la loi de soutien à la formation et aux études (BAföG); les allocations versées aux étudiants sont transformées en prêts à taux zéro. Parallèlement sont adoptées des mesures d'allègement fiscal au bénéfice des entreprises en vue d'encourager l'investissement.

21 mars : Réalignement des parités au sein du SME pour répondre à la crise du franc. Ce dernier est dévalué de $2,5 \%$, tandis que le DM est réévalué de $5,5 \%$.

21-23 novembre : Le Chancelier prend officiellement position devant le Bundestag en faveur du stationnement des fusées américaines à moyenne portée. Le Bundestag se prononce avec une nette majorité pour l'installation des fusées dans les délais prévus. Le ministre fédéral de la Défense, Manfred Wörner, annonce sa mise en œuvre effective à la fin de l'année.

\section{4}

$1^{\text {er }}$ janvier : Nouvelle baisse des prestations sociales, en particulier des prestations d'assurance chômage qui sont réduites de $68 \%$ à $63 \%$ de l'ancien salaire pour les chômeurs célibataires. 
Mars : Adoption d'une loi sur les préretraites prévoyant un départ anticipé à partir de 58 ans, ceci afin de lutter contre le chômage et de favoriser l'insertion de jeunes demandeurs d'emploi. Depuis 1983, et en dépit d'une reprise modérée de la conjoncture, le chômage a franchi la barre des 2 millions et plafonne autour de 2,3 millions de demandeurs d'emploi en 1984-85.

Mai : IG Metall lance la grève dans l'industrie des métaux de Bade-Wurtemberg et de Hesse pour obtenir un nouvel accord cadre (Manteltarifvertrag) prévoyant une réduction de la durée hebdomadaire du travail à 35 heures. IG Druck lance une offensive parallèle dans le secteur de l'imprimerie. A l'issue de conflits de plusieurs semaines et de procédures d'arbitrage, les deux syndicats acceptent un double compromis («Compromis Leber») prévoyant la fixation de la durée conventionnelle de référence à $38 \mathrm{~h} 30$ et une organisation flexible du temps de travail qui peut être modulé entre 37 et 40 heures.

23 mai : Richard von Weizsäcker (CDU), précédemment bourgmestre de Berlin, est élu Président fédéral pour un premier mandat. II sera réélu le 23 mai 1989.

26 juin : Les efforts conjoints entrepris par le président Mitterrand et le chancelier Kohl pour résoudre la crise budgétaire européenne depuis l'échec du sommet de décembre 1983 trouvent leur aboutissement dans le compromis conclu au sommet de juin à Fontainebleau sur la contribution britannique et le financement de la PAC. Ce compromis permet d'engager une relance de la politique d'intégration européenne.

Septembre : Cérémonie de Verdun à la mémoire des morts des deux guerres mondiales au cours de laquelle François Mitterrand et Helmut Kohl se prennent la main en symbole de la réconciliation franco-allemande.

\section{5}

Avril : Augmentation de $0,5 \%$ des cotisations à l'assurance retraite qui passent à $19,2 \%$.

1-6 mai 1985 : Visite du Président Reagan en République fédérale à l'occasion de la réunion du G7 à Bonn et visite au cimetière militaire de Bittburg à l'occasion du $40^{\mathrm{e}}$ anniversaire de la fin de la guerre.

17 juin : Lancement, sur initiative franco-allemande, du projet européen Eureka pour la promotion de la recherche dans les technologies de pointe. Le programme est finalisé lors de la conférence intergouvernementale de Hanovre les 5-6 novembre.

\section{6}

$1^{\text {er }}$ janvier : Première étape de la réforme fiscale. Baisse du taux d'imposition sur le revenu des contribuables des tranches inférieures. La réforme fiscale qui est l'un des éléments clés de la politique économique du chancelier Kohl et qui a été initiée par Gerhard Stoltenberg, ministre fédéral des Finances, sera menée en trois étapes successives $(1986,1988,1990)$. Elle conduira à abaisser tangiblement la pression fiscale directe sur les ménages, ce qui contribuera efficacement à soutenir le retour à la croissance, favorisé par ailleurs par la chute du prix du pétrole, la reprise de l'investissement et de bonnes performances à l'exportation.

17 février : Signature de l'Acte unique européen par lequel les Etats membres décident de réduire par étapes les obstacles à la liberté commerciale intracommunautaire et d'instaurer un marché intérieur européen à l'horizon 1992, ainsi que de renforcer la coopération monétaire européenne. Après ratification par les Etats membres, l'Acte unique entre en vigueur au $1^{\text {er }}$ juillet 1987.

\section{7}

27 Janvier : Elections fédérales. La CDU/CSU subit un recul sensible à 44,3\%, tandis que les libéraux progressent à 9,1\%. Le SPD, mené par Johannes Rau, recule à $37 \%$. Les Verts consolident sensiblement leur position en passant de $5,6 \%$ à $8,3 \%$ des voix et obtiennent 44 sièges au Bundestag. La majorité
Les préretraites au secours du chômage

Offensive pour les 35 heures : compromis sur la flexibilité

Relance de la construction européenne à Fontainebleau

Affirmation du couple franco-allemand

Lancement du programme Eureka

La Réforme fiscale stimule la reprise

Signature de

l'Acte unique européen

Helmut Kohl reconduit pour un second mandat 
Dualisme public/privé dans l'audiovisuel

Rapprochement est-ouest

Du «paquet Delors » au projet d'Union monétaire

Premières réformes structurelles des régimes sociaux

Lancement de l'UEM

Révolution silencieuse en RDA et chute du Mur de Berlin chrétienne-démocrate/libérale est reconduite et réélit Helmut Kohl comme chancelier le 11 mars.

3 avril : Signature par les Länder du Contrat d'Etat sur la radiodiffusion (Rundfunkstaatsvertrag) qui règlemente, sur l'ensemble du territoire de la RFA, le régime de coexistence entre audiovisuel public et privé, conformément au « $4^{\mathrm{e}}$ Jugement sur la radiodiffusion » rendu le 04-11-1986 par le TCF et qui institue un "Ordre dual de la radiodiffusion » (l'existence d'un service public fort est la condition sine qua non de l'existence d'un secteur privé). Ce texte sera amendé à plusieurs reprises (pour la dernière fois en 2008).

Septembre : Visite de Honecker en République fédérale.

8 décembre : Accord Reagan-Gorbatchev à Washington sur le démantèlement des euromissiles.

\section{8}

$1^{\text {er }}$ janvier : Deuxième étape de la réforme fiscale. Baisse du taux d'imposition sur le revenu des tranches moyennes.

11-12 février : Le Conseil européen de Bruxelles adopte les propositions du "Paquet Delors" sur la réforme du budget communautaire, de la PAC et des fonds structurels, mettant ainsi un terme à la crise budgétaire et créant les conditions de la mise en place du marché unique.

27-28 juin 1988 : Le Conseil européen de Hanovre, sous la présidence de Helmut Kohl, décide de préparer le passage à l'Union économique et monétaire européenne et confie à Jacques Delors, président de la Commission, la mission d'en étudier et d'en proposer les étapes pour y parvenir. Une conférence de presse commune de Helmut Kohl et de Jacques Delors souligne l'importance historique du projet.

24-27 octobre : Entretiens entre H. Kohl, H.-D. Genscher et M. Gorbatchev à Moscou.

Novembre-décembre : Première réforme du système de santé en vue de contenir les dépenses de l'assurance-maladie, prévoyant notamment une diminution de certaines prestations, l'augmentation de la participation financière des patients, l'instauration de profils médicaux, une hausse de la cotisation des retraités. Parallèlement, le Ministre fédéral du travail et des affaires sociales, Norbert Blüm, lance le processus d'adoption de la réforme du régime des retraites en vue d'en assurer l'équilibre financier à long terme dans le contexte du vieillissement démographique. La réforme, qui doit s'appliquer à partir de 1992, prévoit notamment de reculer l'âge légal de départ à la retraite de 60 à 63 ans, de calculer les cotisations sur le salaire net, de revoir le mode de calcul des annuités. N. Blüm réussit, au terme de consultations qui se prolongeront toute l'année 1989, à obtenir un consensus des trois grands partis sur cette réforme à long terme qui sera finalement votée le 18-12-1989.

\section{De l'unification de l'Allemagne à l'Union européenne}

\section{9}

12-15 juin : Visite d'Etat de M. Gorbatchev à Bonn. Signature d'une déclaration commune plaçant les relations germano-soviétiques sur de nouvelles bases.

26-27 juin : Le Conseil européen de Madrid approuve les propositions du Rapport de Jacques Delors sur l'UEM qui prévoit sa réalisation en trois étapes et décide de fixer le début de la première étape, celle du renforcement des mécanismes existants du SME, au $1^{\text {er }}$ juillet 1990.

Juillet-septembre : Mouvement d'exode des citoyens de la RDA vers l'ouest via les pays limitrophes de l'est, occupation des ambassades de la RFA, ouverture de la frontière hongroise vers l'Autriche. 
Septembre-octobre : Afflux de réfugiés de RDA. "Manifestations du lundi » à Leipzig et "révolution pacifique » qui mènera à la chute du régime communiste de la RDA.

7 octobre : $40^{\mathrm{e}}$ anniversaire de la RDA en présence de $\mathrm{M}$. Gorbatchev qui apporte un soutien explicite aux réformes. Chute d'Honecker le 17 octobre, Egon Krenz lui succède.

9 novembre : Chute du Mur de Berlin. L'exode à l'ouest d'actifs de la RDA s'amplifie.

17 novembre : La Volkskammer de la RDA élit, sur proposition du SED, Hans Modrow comme nouveau Ministre-président.

28 novembre : Helmut Kohl présente son « Programme en 10 points en vue de surmonter la division de l'Allemagne et de l'Europe " avec comme objectif la réunification de l'Allemagne.

7 décembre : Constitution de la Table ronde (Runder Tisch), instance de concertation entre les différentes composantes de l'opposition réformatrice et les représentants du SED.

Décembre 1989 - janvier 1990 : Processus de réforme et de refondationréunification des anciens partis, émergence de forces politiques nouvelles.

\section{0}

\section{Républlique fédérale}

$1^{\text {er }}$ janvier : Troisième étape de la réforme fiscale. Réaménagement de la progressivité de l'impôt sur le revenu en faveur des classes moyennes supérieures par un élargissement des tranches et une plus grande linéarité des taux. Allègement de la fiscalité en faveur des familles par augmentation échelonnée des forfaits d'exonération par enfant à charge.

28 avril : Le Conseil européen réuni à Dublin approuve le projet d'unification de l'Allemagne.

Mai 1990 : Signature d'accords cadres dans l'industrie des métaux et l'imprimerie prévoyant la réduction par étapes de la durée du travail hebdomadaire de référence à 35 heures à l'horizon 1995 avec possibilité de modulation flexible en fonction de l'activité de l'entreprise.

$1^{\mathrm{er}}$ juillet : Entrée en vigueur de la $1^{\text {ère }}$ étape de l'Union économique et monétaire européenne.

Entrée en vigueur de la Loi sur la structure de la Poste (Poststrukturgesetz) qui réorganise le ministère fédéral des $P \& T$ et constitue la première étape de la privatisation des télécommunications et des services postaux.

\section{République démocratique}

Janvier : Les manifestations populaires en faveur d'une unification rapide prennent de l'ampleur.

28 janvier : Hans Modrow et la Table ronde s'entendent sur la fixation des élections à la Volkskammer au 18 mars. $1^{\text {er }}$ février : Hans Modrow propose un programme d'unification en 4 étapes ; le 7, le gouvernement Kohl propose d'engager immédiatement des négociations sur une union monétaire et une réforme économique. Les 13-14 février se tiennent à Bonn des pourparlers entre les deux gouvernements qui achoppent sur le montant du plan d'aide financière immédiate de la RFA à la RDA.

18 mars : Premières élections libres à la Volkskammer; les partis de l'Alliance pour l'Allemagne favorables à l'unification, à la tête desquels la CDU, frôlent les $48 \%$, tandis que le SPD obtient moins de $22 \%$, le PDS $16,4 \%$, les libéraux $5,28 \%$. Les forces initiatrices de la révolution silencieuse ne totalisent guère plus de $5 \%$. Le 12 avril est constitué, après de longues tractations, un gouvernement de grande coalition Alliance-SPD-Libéraux, qui s'accorde sur le principe d'une unification selon la procédure par adhésion de l'article 23 de la Loi fondamentale. Lothar de Maizières (CDU) est élu Ministre-président.
Modernisation et intégration européenne

«Wir sind ein Volk! »

Elections libres en RDA 
Union monétaire, économique et sociale

Unification politique

Réalisation de l'unité allemande

financier et économique de l'unification

L'alignement problématique des salaires

\section{Vers l'Allemagne unifiée}

5 mai : Début des entretiens 2+4 (RFA-RDA, USA, URSS, France, Grande Bretagne) sur le règlement international de la question allemande.

18 mai : A l'issue de négociations entre les deux gouvernements, un projet de Traité sur la création d'une Union monétaire, économique et sociale est conclu avec entrée en vigueur au $1^{\mathrm{er}}$ juillet.

$1^{\text {er }}$ juillet : Union monétaire, économique et sociale de la RDA et de la RFA qui prévoit : 1/ l'extension et la gestion du DM par la Bundesbank sur le territoire de la RDA selon une parité de 1:1 pour les transactions courantes et pour les avoirs jusqu'à $6000 \mathrm{DM}$, et de 2 : 1 au-delà ; 2/ l'extension des règles de l'économie de marché, c'est-à-dire l'application immédiate de l'ensemble de la législation économique en vigueur et la mise en œuvre de la privatisation des anciennes entreprises d'Etat ; 3/ l'extension à l'Est des droits sociaux et donc la mise en place progressive du système de relations professionnelles, de protection et d'aide sociale.

31 août : Signature du Traité d'unification (Einigungsvertrag) entre les deux Etats.

12 septembre: Signature du Traité sur le règlement final relatif à l'Allemagne (Accord 2+4) qui fixe les frontières définitives de l'Allemagne, impose sa renonciation à l'arme nucléaire et la limitation de ses forces armées, reconnaît son appartenance à l'OTAN, règle les conditions de départ des troupes d'occupation soviétiques. Le 2 octobre, les 4 puissances alliées déclarent à New-York qu'elles reconnaissent la souveraineté de l'Allemagne unie.

3 octobre : En application de l'art. 23 de la Loi fondamentale, la RDA adhère au " territoire où s'applique la Loi fondamentale ", c'est-à-dire à la RFA, et cesse par là même d'exister. Le droit européen communautaire s'applique de facto dans les nouveaux Länder. La ville de Berlin est également réunifiée en tant que Land et capitale de l'Allemagne unie. Le 3 octobre devient la nouvelle fête nationale allemande. La Treuhandanstalt, chargée de la (re)privatisation des entreprises nationalisées par le régime de la RDA, engage ses travaux. Elle sera dissoute au terme de son mandat le 31.12.1994.

14 octobre : Re-décentralisation de l'ex-RDA (entrée en vigueur de la Loi portant création des Länder) et élection des parlements régionaux dans les 5 nouveaux Länder.

2 décembre : Premières élections législatives fédérales de l'Allemagne unifiée. Victoire de la coalition chrétienne-démocrate/libérale sous la direction de Helmut Kohl et Hans-Dietrich Genscher avec une majorité de $54,8 \%$ des voix. Le SPD, sous la conduite d'Oskar Lafontaine, n'obtient que 33,5\%, son plus faible score depuis 1949. L'application séparée de la clause des $5 \%$ dans les Länder de l'Est permet au PDS d'obtenir 17 sièges et à l'Alliance 90/Verts 8 sièges au Bundestag. Ce dernier élit le 17 janvier 1991 Helmut Kohl comme premier chancelier de l'Allemagne unifiée.

\section{1}

28 février : Le chancelier Kohl reconnaît s'être trompé dans son appréciation initiale du coût de l'effort consenti pour l'unification ; désormais, recourir à la dette ne suffit plus, il convient de financer la transformation des nouveaux Länder également par l'impôt. L'impôt de solidarité (Solidaritätszuschlag) de 7,5 \% sur l'ensemble des revenus des ménages et des entreprises est instauré pour un an le 01-07-91, et la TVA passe de $14 \%$ à $15 \%$ le 01-01-1993 (elle sera relevée à $16 \%$ au $1^{\text {er }}$ avril 1998).

8 mars : Adoption du Programme «Aufschwung Ost » d'aide à la reconstruction, à la modernisation des infrastructures et à l'emploi.

Printemps : Accords cadres de branche de revalorisation échelonnée des salaires dans les nouveaux Länder jusqu'en 1995. Ce « rattrapage salarial » programmé en dehors de toute relation avec la situation et l'évolution de la productivité de l'économie est-allemande se révèle beaucoup trop rapide et sera l'une 
des principales causes de la montée du chômage et de la lenteur de la reconstruction des Länder de l'est.

23 avril : Jugement du TFC sur le droit de propriété qui déclare irréversibles les expropriations pratiquées après 1945 en zone soviétique et en RDA mais garantit le droit à indemnisation des anciens propriétaires. Cette clarification débloque le processus de privatisation des entreprises et permet à la Treuhandanstalt de pouvoir procéder aux adjudications en faveur des investisseurs-repreneurs potentiels sans attendre l'identification des anciens propriétaires. La Treuhandanstalt peut désormais se concentrer sur les modalités de la privatisation et opter, selon les cas, en faveur d'une restructuration préalable, à la fois en vue d'attirer les investisseurs potentiels et de préserver l'outil industriel. Au terme du mandat de la Treuhandanstalt fin 1994, le processus de privatisation se soldera par un passif net de 270 milliards DM qui sera inscrit à la dette publique globale dont le remboursement sera étalé sur 40 ans et représentera une charge budgétaire annuelle de 20 milliards DM.

20 juin : Le Bundestag se prononce à une majorité de 18 voix sur 658 votants en faveur de Berlin comme futur siège du Parlement et du gouvernement de l'Allemagne unifiée.

9-11 décembre : Le Conseil européen de Maastricht décide, sous l'impulsion conjointe du Président Mitterrand et du Chancelier Kohl, d'unifier l'ensemble des traités des communautés en traité unique de l'Union européenne et de franchir ainsi une nouvelle étape dans l'intégration de l'Europe.

\section{2}

7 février : Signature du Traité de Maastricht qui crée l'Union européenne. II entre en vigueur le $1^{\mathrm{er}}$ novembre 1993.

2 mai : Signature du Traité sur l'Espace économique européen entre les Etats de la CE et les pays de l'AELE.

18 mai : Départ de Hans-Dietrich Genscher, remplacé par Klaus Kinkel.

$1^{\mathrm{er}}$ juillet : Entrée en vigueur de la réforme de la procédure du droit d'asile qui devient plus restrictive pour endiguer le flot croissant de « réfugiés économiques ».

21 décembre : Modification de la Loi fondamentale. L'art. 23 qui avait permis la réunification est supprimé et remplacé par une nouvelle version permettant les transferts de souveraineté de la RFA vers l'UE prévus par le Traité de Maastricht et inscrivant dans une même continuité le processus d'intégration européenne et I'Unité allemande. II associe plus étroitement les Länder à la politique européenne (pouvoirs de co-décision accrus dans les domaines relevant de leur compétence).

\section{L'Allemagne unie entre intégration européenne et globalisation}

1993

$1^{\mathrm{er}}$ janvier : Entrée en vigueur du Marché unique européen.

3 mars : Pacte de Solidarité pour le financement de l'unité allemande adopté par le gouvernement fédéral, les ministres-présidents des Länder et les dirigeants des trois grands partis (dont le SPD) qui prévoit un ensemble de mesures de soutien au bénéfice des nouveaux Länder. Réinstauration pour une durée indéterminée de l'impôt solidarité à compter du 01-01-1995.

Printemps : La récession mondiale, conjuguée aux restructurations auxquelles l'industrie allemande doit faire face dans le contexte de la globalisation, frappe brutalement une économie allemande qui se trouve en pleine expansion mais dont les charges ont considérablement augmenté sous l'effet de l'unité. La récession révèle la nécessité d'une restauration de la compétitivité et de l'attractivité du site allemand de production (Standort Deutschland).
Droit de propriété et privatisation

Le choix de Berlin

Sommet de Maastricht

Traité de l'Union européenne

Réforme du droit d'asile

Réforme de l'article 23 de la Loi fondamentale

Pacte de solidarité pour l'unité allemande

Récession économique 
Emergence d'une politique allemande de compétitivité globale

Accord Volkswagen : la RTT au service du maintien des emplois

Réforme structurelle du système de santé

Réforme des chemins de fer

Libéralisation et privatisation des télecommunications et des services postaux

Elections au Bundestag

Quatrième mandat pour Helmut Kohl

Intégration des nouveaux Länder dans le fédéralisme financier
Septembre : Le gouvernement fédéral publie un rapport sur la « Préservation de l'avenir du site Allemagne » (Zukunftssicherung des Standorts Deutschland) où il développe une nouvelle approche de la compétitivité globale, fondée sur la convergence des politiques économiques publiques autour d'une stratégie collective de renouveau et de la promotion d'une culture favorable au changement. Adoption, le 19-09-1993, d'une loi visant à garantir l'attractivité du site Allemagne (Standortsicherungsgesetz). Son objectif est de préserver la compétitivité internationale globale du site grâce à l'amélioration des conditions cadre réservées aux activités. Elle comporte principalement un ensemble de mesures d'allègement fiscal en faveur des entreprises qui entrent en vigueur au 01-01-1994.

Décembre : Dans le cadre d'un plan de restructuration, la direction de VW et IG Metall signent un accord d'entreprise permettant le maintien de l'emploi contre une réduction-flexibilisation du temps de travail à un plancher de 28,8 hebdomadaires, assortie d'une réduction de salaire de $12 \%$. Cet accord inaugure une série d'accords ou «pactes pour l'emploi » passés dans différentes branches, notamment celle des métaux, dans les années 1994-95, par lesquels la réduction et la flexibilité négociées du temps de travail sont utilisées aux fins de maintenir les emplois en période conjoncturelle difficile.

20 décembre : Amendement de la Loi fondamentale en prévision de la privatisation des chemins de fer fédéraux.

21 décembre : Adoption de la Loi structurelle sur la Santé (Gesundheitsstukturgesetz), initiée par le ministre fédéral de la santé, Horst Seehofer, et dont l'objectif principal est d'enrayer la dérive inquiétante des dépenses de santé. Elle instaure une politique de budget global pour toutes les catégories de soins et les médicaments, une régulation de l'installation des médecins de caisse en fonction de la densité de l'offre, une augmentation de la participation des assurés au coût des soins. La loi entre en vigueur au 01-01-1994 et se heurtera à une forte résistance des professions médicales.

\section{4}

$\mathbf{1}^{\mathrm{er}}$ janvier : Début de la deuxième étape vers l'Union économique et monétaire.

Deutsche Bahn AG naît par fusion des sociétés publiques est- et ouest-allemandes. Entrée en vigueur de la Loi sur la réorganisation des chemins de fer (Eisenbahnneuordnungsgesetz) qui prévoit quatre axes de réformes : désendettement et désétatisation/privatisation de l'administration publique des chemins de fer, libéralisation du marché et transfert aux Länder des compétences en matière de transports en commun et de liaisons régionales.

29 juin : Modification de la Loi fondamentale en prévision de la libéralisation des télécommunications et des services postaux : levée du monopole législatif et administratif de l'Etat fédéral. Puis transformation du ministère fédéral des P\&T en trois sociétés par actions: Deutsche Telekom, Deutsche Postbank et Deutsche Post (Loi sur la réorganisation des postes : Postneuordnungsgesetz; entrée en vigueur le 01-01-1995).

16 octobre : Elections fédérales. Résultats mitigés pour la coalition chrétiennelibérale : la CDU n'obtient que $41,5 \%$ des voix et le FDP 6,9\%, ce qui ne lui donne qu'une majorité de 10 sièges. Le SPD, sous la conduite de R. Scharping, remonte à $36,4 \%$, tandis que les Verts deviennent le $3^{\mathrm{e}}$ parti avec $7,6 \%$ des voix, le PDS ne restant représenté au Bundestag avec $4,4 \%$ des voix qu'à la faveur de l'obtention de 4 mandats directs dans la partie est de Berlin.

17 novembre : $\mathrm{H}$. Kohl est réélu chancelier pour la $5^{\mathrm{e}}$ fois et entame son $4^{\mathrm{e}}$ mandat.

\section{5}

$1^{\mathrm{er}}$ janvier : Entrée en vigueur de la réforme du fédéralisme financier qui intègre les nouveaux Länder dans le système de péréquation entre les collectivités territoriales et crée le Pacte de solidarité (Solidarpakt I), destiné à la reconstruction de l'économie des nouveaux Länder et doté de 105 milliards € sur la période 1995-2004. 
Mise en place de l'assurance-dépendance (Pflegeversicherung) créée par la loi du 26-05-1994 en tant que cinquième branche du système de protection sociale et financée par un supplément de cotisation de $1 \%$, puis de $1,7 \%$ du salaire plafonné.

\section{6}

Printemps : La CDU obtient des scores médiocres aux élections régionales, ce qui a pour effet de réduire son influence au Bundesrat et la capacité du gouvernement Kohl à poursuivre ses projets de réforme.

Juillet : Le « Programme d'action pour l'investissement et l'emploi » présenté par le gouvernement et qui comporte d'importantes mesures d'économie dans le domaine social est bloqué par le Bundesrat.

Mi-septembre : Il est repris en partie dans la loi portant sur les mesures d'économies (dite "Sparpaket ») qui prévoit notamment de réduire les indemnités maladie et les compléments de salaires (heures supplémentaires, primes et avantages contractuels divers) pris en compte dans le maintien de la rémunération en cas de maladie. Cette remise en cause des acquis dans le dispositif de la Lohnfortzahlung suscite le mécontentement des partenaires sociaux. Par ailleurs, la protection contre le licenciement est assouplie pour les petites entreprises et la durée maximale des CDD portée de 18 mois à 2 ans.

18 novembre : Entrée en bourse de Deutsche Telekom AG. L'ouverture du capital de l'opérateur historique aux petits épargnants est le premier recours à l'actionnariat populaire depuis les années du miracle économique et un élément important de la politique de modernisation de la place financière allemande.

\section{De la paralysie réformatrice au réformisme social-écologique}

\section{7}

26 avril : Alors que l'Allemagne s'enfonce dans ce que les observateurs étrangers nomment la « germanosclérose », le président Roman Herzog (CDU), élu en 1994, tient à Berlin (Hôtel Adlon) un discours où il tente d'impulser un " sursaut » réformateur dans la société.

26 juin : Les projets de réforme fiscale du gouvernement Kohl qui prévoient une baisse substantielle des taux d'imposition de l'impôt sur le revenu et de l'impôt sur les bénéfices des entreprises sont adoptés par le Bundestag, mais échouent finalement en commission de conciliation devant l'opposition majoritaire du Bundesrat menée par les Länder dirigés par le SPD.

Novembre : Le Conseil des Sages consacre son rapport annuel 1997/98 à l'impératif d'une politique de réformes pour redynamiser la compétitivité de l'économie allemande dans un monde globalisé. II y formule une série de recommandations reposant toutes sur une "politique de l'offre " consistant à créer un environnement favorable à l'activité, c'est-à-dire orientée sur le long terme et associant l'ensemble des acteurs économiques et politiques.

11 décembre : Le gouvernement fait adopter au parlement trois mesures importantes : l'ouverture à la concurrence du marché des télécommunications et l'institution d'une autorité de régulation sectorielle pour les télécommunications et les services postaux: la Regulierungsbehörde für Post und Telekommunikation (entrée en vigueur : 01-01-1998) ; la Loi de réforme de l'assurance retraite (Rentenreformgesetz) qui prévoit, à compter de 1999, la prise en compte du facteur démographique dans le calcul des retraites et induit la réduction progressive du taux de pension de $70 \%$ à $64 \%$ (cette disposition sera annulée par le gouvernement Schröder); et le relèvement de la TVA de $15 \%$ à $16 \%$ au $1^{\mathrm{er}}$ avril 1998 (NB : l'impôt solidarité avait été réduit le $1^{\text {er }}$ octobre de $7,5 \%$ à $5,5 \%$ avec effet au 01-01-1998).
Création de

l'assurance-dépendance

Montée des mécontentements face à la réduction des acquis sociaux

Deutsche Telekom entre en bourse

Paralysie réformatrice du gouvernement Kohl

Les Sages relancent la Standortpolitik

Derniers feux pour Helmut Kohl 
Vers la monnaie unique

L'alternance rouge-verte

Pacte pour l'emploi

Exit Lafontaine

Création de l'écotaxe

Les dérives du fédéralisme financier en ligne de mire de Karlsruhe

\section{8}

1-2 mars : Au lendemain de sa victoire aux élections de Basse-Saxe, Gerhard Schröder est désigné candidat Chancelier par le SPD.

23 avril : Sur proposition du gouvernement fédéral, le Bundestag puis le Bundesrat approuvent la participation de la RFA au passage à la $3^{\mathrm{e}}$ phase de l'UEM et à la création de l'Euro au $1^{\text {er }}$ janvier 1999.

1-3 mai : Le Conseil européen extraordinaire de Bruxelles décide de la création de l'Euro comme monnaie unique au $1^{\mathrm{er}}$ janvier 1999.

30 juin : La Banque centrale européenne débute ses activités à Francfort sur le Main.

Septembre-novembre : Elections au Bundestag. La coalition CDU-FDP perd les élections et Helmut Kohl quitte le pouvoir au terme d'une longévité gouvernementale sans précédent dans I'histoire allemande. Le chancelier Gerhard Schröder (SPD) forme un gouvernement de coalition SPD/Verts, dans laquelle Oskar Lafontaine joue un rôle déterminant à la fois comme président du SPD et comme Ministre des finances aux attributions économiques renforcées. Les Verts entrent au gouvernement fédéral. Joschka Fischer devient vice-chancelier, ministre des Affaires extérieures. Les Verts prennent également en charge le ministère de l'Environnement (Jürgen Trittin).

7 décembre : Mise en place, à l'initiative du Chancelier, du « Pacte pour l'emploi, la formation et la compétitivité» ( « Bündnis für Arbeit »), instance de dialogue tripartite gouvernement-patronat-syndicats ayant pour objectif de promouvoir une stratégie concertée de lutte contre le chômage, placée au rang de priorité $\mathrm{n}^{\circ} 1 \mathrm{du}$ gouvernement. Le pacte se limitera à une série de rencontres au sommet suivies de déclarations communes mais ne débouchera pas sur des engagements concrets des différents acteurs et se soldera finalement par un échec.

\section{9}

$\mathbf{1} €=\mathbf{2} \mathrm{DM} \quad \mathbf{1}^{\mathrm{er}}$ janvier : Entrée en vigueur de l'UEM après fixation irrévocable, le 31 décembre 1998, de la parité de l'Euro par rapport aux autres monnaies. La parité $\mathrm{DM} / €$ est fixée à $1: 1,95583$ - en fait, dans l'esprit des Allemands, à $1: 2$. L'Euro entre en usage comme monnaie scripturale au sein de la zone Euro.

4 mars : Adoption de la réforme fiscale (Steuerentlastungsgesetz) qui prévoit un allègement de l'impôt sur le revenu et les bénéfices en trois étapes, échelonnées sur 1999, 2000, 2003. Elle comporte à la fois des élargissements d'assiette (notamment vers les bas revenus) et des baisses de taux d'imposition aux tranches extrêmes. Ce premier réaménagement fiscal est jugé décevant par les acteurs économiques.

11 mars : Démission d'Oskar Lafontaine à la suite de ses prises de position en faveur d'une politique économique « mixte » de soutien à la croissance et à l'emploi fondée sur le desserrement de la rigueur budgétaire et la baisse des taux d'intérêt. Cet affichage, qui est perçu comme une mise en cause à la fois de la politique de la BCE et de son indépendance, suscite de vives critiques dans les milieux économiques et conduit à un conflit ouvert avec le Chancelier. Lafontaine démissionne de la présidence du SPD et de ses fonctions ministérielles. Hans Eichel lui succède à la tête d'un ministère des finances "normalisé » et met en œuvre une politique systématique et difficile d'économies budgétaires.

$1^{\text {er }}$ avril : Entrée en vigueur progressive jusqu'au 01-01-2003 de la Loi sur l'entrée dans la réforme écologique de la fiscalité (Gesetz zum Einstieg in die ökologische Steuerreform) du 24-03 qui instaure une écotaxe assise sur la consommation d'énergie et dont le double objectif est d'abaisser les cotisations retraite "pour rendre le facteur travail moins cher » et de financer le développement des énergies renouvelables.

11 novembre : Jugement du TCF sur le fédéralisme financier. II fait injonction aux législateurs de redéfinir les modalités du système de solidarité financière entre les collectivités territoriales en respectant le principe de « l'interdiction du ni- 
vellement » (Nivellierungsverbot) qui découle du principe constitutionnel de l'équité des chances. A la suite de cet arrêt, le mode de calcul de la péréquation financière au profit des nouveaux Länder devra être revu.

24 novembre : Plan de sauvetage du groupe BTP Philipp Holzmann, mis en place à l'instigation du chancelier Schröder avec le concours des banques et d'aides publiques. L'opération suscite de vives critiques dans les milieux économiques.

Novembre-décembre: Adoption par le parlement du premier programme d'économies («Sparpket») présenté par H. Eichel, qui instaure au 01-01-2000 une baisse de la contribution de l'Etat à l'assurance retraite et un plafonnement en pouvoir d'achat des pensions pour 2000/01, une réduction de la durée du service militaire de 13 à 11 mois et diverses mesures en faveur des familles ayant des enfants.

\section{0}

4 juin : Adoption d'un consensus sur la sortie progressive du nucléaire, négocié par la coalition fédérale (SPD/Verts) et les principaux électriciens. II est suivi, le 14-12-2001, par le vote d'une loi réglementant les étapes progressives de l'abandon de la production d'électricité à partir du nucléaire. Entrée en vigueur le 26-042002, elle limite notamment la durée de vie des centrales existantes à 32 ans.

23-24 mars : Lors du Conseil européen à Lisbonne, les Etats membres décident d'une stratégie de modernisation de leurs économies pour "faire de l'Europe l'économie la plus compétitive dans l'ère du savoir " Cette "Stratégie de Lisbonne », connue aussi sous le nom de politique « pour la croissance et l'emploi », prévoit non seulement un effort conséquent d'innovation (3\% du PIB consacré à la R\&D à l'horizon 2010), mais aussi d'importantes réformes structurelles, notamment en matière de protection sociale.

14 juillet : Adoption de la Loi de réduction fiscale (Steuersenkungsgesetz) et d'une série d'allégements fiscaux spécifiques aux PME. Les dispositions de cette réforme du code allemand des impôts visent ouvertement à soutenir la compétitivité de l'économie par une baisse par étapes du poids des prélèvements, à la fois de l'impôt sur le revenu (relèvement du seuil d'imposition, réduction du taux maximal à $48,5 \%$, puis $43 \%$ en 2005) et de l'impôt sur les bénéfices (taux unique à $25 \%$ ). L'avoir fiscal est instauré sur les dividendes, et les plus-values sur cessions de valeurs mobilières sont entièrement exonérées d'impôt à compter de 2002, ceci en vue de dynamiser la place financière allemande. En dépit de la résistance initiale de l'opposition, Hans Eichel obtient l'approbation du Bundesrat sur sa réforme.

\section{Retour au réalisme sous la pression de la crise}

\section{1}

$1^{\text {er }}$ janvier : Basculement à l'euro dans les 15 Etats membres concernés. L'Allemagne abandonne le Deutsche Mark.

19-21 mars : Congrès constituant du nouveau syndicat unitaire des services: Vereinigte Dienstleistungsgewerkschaft (ver.di). Ce conglomérat de branche naît, après cinq ans de négociations, de la fusion de cinq syndicats : ÖTV (services publics et transports), DAG (employés), Deutsche Postgewerkschaft (services postaux), HBV (commerce, banque et assurance) et IG Medien.

Mai : Réforme des retraites, qui entre progressivement en vigueur à partir du 0101-2002. Elle apporte un changement important de doctrine : pour compenser la réduction à terme des prestations du régime légal d'assurance retraite induite par le vieillissement démographique, la Loi sur la formation d'un patrimoine vieillesse (Altersvermögens-Gesetz) encourage le recours accru à la capitalisation par la mise en place d'une épargne retraire subventionnée (« retraites Riester »). L'objectif de la réforme, initiée par le ministre du Travail, Walter Riester (ancien prési-
Eichel entre en scène

Sortie du nucléaire

Stratégie de Lisbonne

Réforme fiscale version Eichel

Entrée en vigueur de l'euro

Constitution du syndicat ver.di

Réforme Riester : retraite par capitalisation 
Conseils d'entreprise

Nouveau pacte de solidarité en faveur de l'Est

Réforme de la Bundesanstalt für Arbeit

Pacte national de stabilité

Commission Hartz

Courte victoire et second mandat pour Gerhard Schröder

Eichel colmate les brèches dent d'IG Metall) est de rééquilibrer les charges entre les trois piliers du système allemand d'assurance vieillesse : assurance retraite légale (80\% des assurés), retraites d'entreprise et prévoyance complémentaire de la fonction publique $(10 \%)$, et prévoyance individuelle $(10 \%)$. La même réforme crée un 'minimum vieillesse' destiné aux personnes en difficulté (Gesetz über eine bedarfsorientierte Grundsicherung im Alter und bei Erwerbsminderung) qui entre en vigueur le 0101-2003.

22 juin : Réforme du Betriebsverfassungsgesetz. De nouvelles dispositions facilitent la constitution des Conseils d'entreprise (notamment dans les PME) et renforcent leurs droits de co-décision en matière de formation, de protection de l'environnement et de promotion des femmes.

5-13 juillet : Accord entre Fédération et Länder sur le plafonnement de la base de péréquation financière entre Länder (ramenée de $80 \%$ à $72,5 \%$ de leur produit de TVA) et prolongement dégressif du Pacte de solidarité financière au profit des nouveaux Länder (Solidarpakt II) de 2005 jusqu'en 2019, à hauteur d'un volume global de transfert de 156 milliards $€$ (pour la période 1990-2000, l'effort global de solidarité en faveur de l'est - Aufbau Ost - a été de 500 milliards $€$ ).

\section{2}

A la suite de la crise boursière liée à l'éclatement de la bulle spéculative sur les valeurs technologiques, l'économie des pays industriels connaît un net ralentissement dont l'économie allemande, largement dépendante de l'exportation, accuse le contrecoup. Sous l'effet d'un niveau de charges trop élevé qui pèse sur sa compétitivité externe et étouffe sa demande intérieure, et des rigidités de son système social qui retardent son adaptation, l'Allemagne s'enfonce dans le marasme. Avec une croissance zéro en 2003 et un recul de $-0,2 \%$ de son PIB en 2003, elle fait alors figure de «lanterne rouge » de l'Europe et ne parvient à endiguer ni la montée du chômage, ni celle des déficits.

27 mars : A la suite de la révélation du maquillage pratiqué sur les statistiques relatives au placement des chômeurs, Walter Riester engage une restructuration de l'Office fédéral du travail.

6 juin : En vue de réduire la montée de l'endettement et d'empêcher que le déficit public global ne franchisse la norme européenne des $3 \%$ du PIB, la Fédération et les Länder s'engagent sur l'objectif conjoint d'une limitation des dépenses pour 2003 et 2004.

Eté : Remise du rapport de la Commission présidée par Peter Hartz, Directeur des affaires sociales de VW, chargée par G. Schröder d'établir un ensemble de propositions pour moderniser les services et le dispositif d'aide à l'emploi aux fins d'améliorer la fluidité du marché du travail. Ce rapport servira de base à la préparation d'une série de lois dites "Lois Hartz ».

2 septembre : Elections fédérales. G. Schröder l'emporte de 7000 voix sur Edmund Stoiber (CSU). La coalition SPD-Verts se maintient, grâce à une progression des Verts de $+1,9 \%$ (à 8,6 \%), avec une courte majorité de 7 sièges.

16 novembre : Après de longues discussions, la coalition SPD-Verts adopte un programme gouvernemental qui conjugue la priorité de la lutte contre le chômage avec la promotion d'un cadre général des activités économiques «prenant en compte la dimension écologique et sociale ». G. Schröder avait été réélu chancelier le 22-10 à la tête d'un gouvernement dans lequel un grand ministère de l'Economie et du Travail, confié à Wolfgang Clement, ministre-président de Rhénanie du Nord-Westphalie, occupe une place centrale.

15 novembre : Devant l'aggravation inattendue de la situation budgétaire, et invoquant des raisons conjoncturelles exceptionnelles, Hans Eichel fait voter pour 2002 un collectif budgétaire dont l'endettement final de 35 milliards $€$ dépasse la norme constitutionnelle. Dans la foulée, le Bundestag adopte un plan d'assainissement d'urgence comportant des mesures d'économies et une hausse des cotisations sociales pour 2003. 


\section{Le tournant de l'Agenda 2010 : cap tardif vers les réformes structurelles}

2003

$1^{\text {er }}$ janvier : Entrée en vigueur, après leur adoption le 20-12-2002, des deux premières Lois pour des services modernes du marché de l'emploi (Gesetze für moderne Dienstleistungen am Arbeitsmarkt), qui prévoient respectivement la mise en place, au sein des offices, d'agences de placement temporaire au bénéfice des demandeurs d'emploi, ainsi que la possibilité offerte à ces derniers d'opter pour un statut aidé d'auto-entrepreneur (Ich-AG) ou pour des Mini-Jobs rémunérés. Ces mesures visent à encourager le chômeur à se prendre en charge et à se réinsérer. La $3^{\mathrm{e}}$ loi, entrée en vigueur au $1^{\mathrm{er}}$ avril, transforme la Bundesanstalt en une Agence fédérale pour l'emploi (Bundesagentur für Arbeit) à l'intérieur de laquelle les services locaux sont transformés en Jobs-center intégrant l'ensemble des services aux demandeurs.

14 mars : Alors que l'Allemagne semble solidement installée dans le rôle de " lanterne rouge de l'Europe ", le chancelier Gerhard Schröder reconnaît, dans le discours de politique générale qu'il tient au Bundestag six mois après sa réélection, que la crise économique que traverse l'Allemagne est due avant tout à des raisons structurelles internes. S'inscrivant pleinement dans la Stratégie de Lisbonne et invoquant la responsabilité particulière de l'Allemagne dans la croissance de l'UE, il propose un ambitieux programme de réformes économiques et sociales : "l'Agenda 2010 » qui doit replacer l'Allemagne au premier rang pour l'emploi et le niveau de vie. Au-delà de la politique d'activation du marché du travail engagées par les lois Hartz, priorité est donnée à une réforme d'ensemble du système de protection sociale et à la baisse des prélèvements. L'Agenda reçoit un accueil défavorable au sein des syndicats et suscite des remous au sein du SPD qui, sous la menace de la démission du Chancelier, l'approuve à son congrès extraordinaire le $1^{\mathrm{er}}$ juin.

20 mars : Dans une allocution télévisée, le Chancelier condamne officiellement la guerre en Irak.

Septembre : Projet de loi sur la réforme de l'assurance maladie, soutenu également par l'opposition.

Décembre : Projet de loi sur la soutenabilité des retraites et avancement du calendrier de mise en œuvre de la réforme fiscale.

\section{4}

$1^{\text {er }}$ janvier : L'UE 15 accueille 10 nouveaux Etats membres est-européens, cet élargissement scellant la réunification de l'Europe. Elle s'élargira à 27 membres avec la Bulgarie et la Roumanie au 01-01-2007.

Printemps : IG Metall et Gesamtmetall signent l'Accord de Pforzheim qui permet aux entreprises de la branche du travail des métaux de déroger aux mimima de branche, non plus seulement en cas de difficultés, mais désormais aussi pour des impératifs de compétitivité. II avait été précédé d'un accord-pilote conclu dans le Bade-Wurtemberg au printemps 2002, qui étendait sur l'ensemble du territoire la possibilité pour les entreprises en difficulté de bénéficier des clauses d'ouverture leur permettant de ne pas appliquer les hausses salariales conclues dans la branche, une possibilité jusqu'ici réservée aux entreprises dans les nouveaux Länder. L'accord de Pforzheim a ouvert la voie à une série d'accords sur la hausse de la durée hebdomadaire du travail conclus à l'été 2004 (DaimlerChrysler, Opel, Siemens, VW). II marque la fin de la doctrine du partage du travail concrétisée jusque-là par les 35 heures dans la métallurgie et la nouvelle politique de flexibilité du syndicat IG Metall. Conjugués à la modération salariale dont font preuve les syndicats depuis la fin des années 1990, les accords de flexibilité con-

Agenda 2010

Offensive réformatrice au Parlement

Elargissement de l'UE à l'est

Flexibilité négociée et compétitivité 
Hartz IV : remise à plat de l'indemnisation chômage

Convergence franco-allemande pour assouplir le Pacte de stabilite

Impopularité dans l'électorat social-démocrate et défaites électorales

Question de confiance et dissolution du Bundestag

Elections fédérales anticipées tribuent efficacement à la restauration de la compétitivité des entreprises allemandes et à leurs excellents résultats à l'exportation.

\section{5}

$1^{\text {er }}$ janvier : Adoptée avec retard le 01-07-2004, après de longs débats et suivie de mouvements de protestation dans les nouveaux Länder, la loi Hartz IV entre en vigueur. Elle a pour objectif d'inciter à un retour rapide à l'emploi, en faisant en sorte qu'au-delà du versement des allocations d'assurance chômage liées à l'activité antérieure (et dont la durée a été abaissée à 12 mois en règle générale), le versement d'une indemnité d'assistance (Arbeitslosengeld II) soit conditionné par une recherche active d'emploi. Le montant de cette dernière est forfaitaire et aligné sur celui de l'aide sociale (minimum vital réservé aux personnes en mal d'insertion sociale). Difficile à appliquer du fait d'un certain nombre d'imprécisions dans sa rédaction, cette loi a donné lieu à d'innombrables contestations. Combinée aux autres mesures incitatives de réinsertion et d'emploi aidé, elle a néanmoins efficacement contribué à la diminution du nombre de chômeurs et à la remontée du taux d'activité, soulageant ainsi la dérive financière du système de protection contre le chômage.

20 mars : Conjointement avec son collègue français, Thierry Breton, Hans Eichel obtient, lors du sommet européen de Bruxelles, un assouplissement de l'application des critères du Pacte européen de stabilité. L'Allemagne sera autorisée à déduire les aides aux nouveaux Länder et la contribution allemande au budget de l'UE dans le calcul du déficit public global.

12 mai : Le Bundestag ratifie le Traité constitutionnel européen par 569 voix contre 23. Le Bundesrat y procède le 27.

20 février : Le SPD perd les élections et échoue à se maintenir au gouvernement dans le Land de Schleswig-Holstein qu'il dirigeait depuis 1987. Les débats internes avec la gauche du parti et avec l'aile syndicale se font virulents.

22 mai : Seconde et sévère défaite électorale du SPD dans son fief électoral de Rhénanie du Nord-Westphalie, le Land le plus peuplé d'Allemagne, dont il détenait la direction du gouvernement depuis 1966 (et depuis 1995 à la tête d'une coalition avec les Verts). Avec 37,1\% des voix, il obtient son plus mauvais résultat depuis 1954.

Au soir de cette défaite, le Chancelier déclare ne plus avoir de majorité pour conduire sa politique de réforme, et en accord avec Franz Müntefering, président du parti, il fait part de son intention de provoquer des élections fédérales anticipées.

$\mathbf{1}^{\mathrm{er}}-\mathbf{2 7}$ juillet : G. Schröder pose la question de confiance devant le Bundestag au motif qu'il ne dispose plus ni dans sa majorité ni au Bundesrat de soutiens politiques suffisants pour gouverner. La confiance lui est refusée. Le Président Horst Köhler prononce la dissolution du Bundestag le 27 et fixe la date des élections anticipées au 18 septembre. Le TCF valide la procédure le 25-08.

11 juillet : L'autorité de régulation sectorielle ( $P \& T)$ est rebaptisée Agence fédérale des Réseaux (Bundesnetzagentur) et voit ses pouvoirs étendus au secteur de l'énergie ; ils sont élargis au secteur ferroviaire le 01-01-2006.

17 juillet : Le PDS se transforme en Linkspartei, et ouvre ses listes électorales aux dissidents de la gauche social-démocrate et syndicale réunis dans la WASG et parmi lesquels figure Oskar Lafontaine.

18 septembre : A l'issue d'une remarquable campagne électorale, G. Schröder fait, avec 34,2 \% des voix pour le SPD, jeu presque égal avec sa rivale, Angela Merkel, pourtant largement donnée favorite. Celle-ci n'obtient que 35,2 \% pour la CDU/CSU, et son projet d'alliance avec les Libéraux (9,8 \%) n'a donc pas été ratifié. Fort de ce résultat et du maintien des Verts $(8,1)$, Schröder revendique un moment sa possible reconduction, mais doit y renoncer devant la possibilité offerte à son parti de rester au gouvernement au sein d'une grande coalition répondant aux attentes du corps électoral. 


\section{Les deux grands partis s'allient pour restaurer l'équilibre et la croissance}

18 novembre : Signature du contrat de coalition entre le SPD, la CDU et la CSU, après approbation par les congrès respectifs des partis. Points clés du programme gouvernemental : soutien à l'innovation et à la croissance par l'investissement, assainissement budgétaire et financier, rééquilibrage et réforme de la fiscalité, assouplissement du marché du travail et baisse des cotisations chômage, poursuite de la réforme des retraites, dynamisation de la politique familiale, réforme du fédéralisme.

22 novembre : Le Bundestag élit Angela Merkel chancelière fédérale par 397 voix contre 202 et 12 abstentions. Son gouvernement comprend 5 ministres CDU, 2 de la CSU et 8 du SPD, dont le vice-chancelier Hans Müntefering, ministre du Travail et des Affaires sociales, le ministre des Finances, Peer Steinbrück, et le ministre des Affaires extérieures Frank-Walter Steinmeier. Les 3 femmes ministres du SPD, issues du gouvernement précédent, sont reconduites dans leurs fonctions.

30 novembre : Dans sa déclaration de politique générale, Angela Merkel présente les différents volets du programme gouvernemental qu'elle résume sous le triptyque " assainir, réformer, investir » et dont l'objectif central est de "refaire de l'Allemagne, à moyen terme, le moteur de l'Europe ».

\section{6}

19 mai-16 juin : Le parlement approuve la revalorisation de la TVA de 16 à $19 \%$ au $1^{\mathrm{er}}$ janvier 2007. Les recettes nouvelles seront consacrées pour les deux tiers à la réduction du déficit budgétaire, pour un tiers à l'abaissement des cotisations chômage de $6,5 \%$ à $4,5 \%$. Parmi d'autres mesures budgétaires figurent la suppression d'abattements fiscaux pour frais de déplacement professionnels ainsi que la suppression de la subvention de l'Etat fédéral à la Bundesagentur für Arbeit.

$1^{\text {er }}$ juillet : Après une évaluation indépendante de l'application des Lois Hartz réalisée en février, le gouvernement opère une série d'ajustements dans le dispositif, principalement dans Hartz IV avec l'alignement de l'aide forfaitaire de base $A L G$ II en vigueur à l'est sur le montant de l'ouest ( $345 €)$ et diverses réductions de prestations dont l'impact financier s'est révélé trop coûteux, notamment la suppression de l'allocation logement pour les moins de 25 ans.

30 juin-7 juillet : Bundestag et Bundesrat procèdent à la réforme politique du fédéralisme en redéfinissant et en clarifiant la répartition des compétences exclusives respectives entre Bund et Länder. Le pouvoir de contrôle des Länder sur les lois fédérales via le Bundesrat est renforcé dès lors que celles-ci impliquent des charges financières pour leur budget. En contrepartie, les Länder deviennent co-responsables, à hauteur de $35 \%$, des sanctions possibles pour le non-respect de la discipline budgétaire du pacte de stabilité européen.

19 octobre : Dans un arrêt (affaire Berlin vs. Bund), le TCF réaffirme le " principe selon lequel l'autonomie politique des Länder implique que ceux-ci en gèrent, de manière autonome et responsable, les conséquences budgétaires ». Ce jugement contribue à nourrir les négociations sur la réforme du fédéralisme financier et l'adoption de règles de stabilité budgétaire pour les Länder.

15 décembre : Bundestag et Bundesrat engagent le processus de réforme du fédéralisme financier en instituant une commission mixte de 32 membres chargée de redéfinir les relations financières entre Bund et Länder (clés de répartition des recettes fiscales, financements conjoints, règles de péréquation financière, pacte de stabilité interne à la RFA) qui avaient été pour l'essentiel définies au temps de la Grande coalition des années 1966-1969.

Septembre-novembre : Mise en place, au 01-01-2007, de l'allocation de congé parental (Elterngeld) au profit d'un parent qui interrompt son activité pour se
Grande Coalition SPD-CDU/CSU

Angela Merkel, première chancelière allemande

Refaire de l'Allemagne le moteur de l'Europe

Hausse de la TVA de 3 points

Réajustement des lois Hartz

Réforme du fédéralisme

Revalorisation de la politique familiale 
Nouveau recul de l'âge de départ à la retraite

Réorganisation de l'assurance maladie

Agenda climatique 2020

Réforme de la fiscalité des entreprises

Nouvelle avancée de la politique familiale

Une consolidation budgétaire et financière réussie consacrer à la garde d'un enfant nouveau-né. L'allocation est versée pendant 12 mois, avec 2 mois supplémentaires possibles pour le conjoint, à hauteur de $67 \%$ du revenu net dans la limite de $1800 €$.

\section{7}

Janvier-juin : Présidence allemande du Conseil de l'Union et du G8. Angela Merkel fait ses preuves sur la scène européenne et internationale en faisant adopter au Conseil de l'UE de mars un plan d'action pour la sauvegarde de l'environnement et des orientations pour une politique énergétique commune, puis en dénouant au sommet du 23 juin à Bruxelles le blocage sur la réforme du Traité de I'Union. Au sommet du G8 à Heiligendamm, le 6 juin, elle obtient un accord sur l'objectif de la réduction de moitié des émissions de gaz à effet de serre à l'horizon 2050.

9 mars : Décision du Bundestag de reculer progressivement l'âge légal de départ à la retraite de 65 à 67 ans de façon échelonnée de 2012 à 2029. Après 3 ans de gel des pensions, et une augmentation du taux de cotisation de $19,5 \%$ à $19,9 \%$ au 01-01-2007, les retraites sont revalorisées de 0,54 \% au 01-07. Elles le seront à nouveau de $1,1 \%$ en 2008 .

$1^{\mathrm{er}}$ avril : entrée en vigueur de la Loi de renforcement de la concurrence dans l'assurance maladie (GKV-Wettbewerbsstärkungsgesetz). La réforme modifie en profondeur l'organisation de l'assurance maladie : elle instaure une concurrence accrue entre les caisses en aval au niveau de l'offre de soins et de la prise en charge des prestations, tandis qu'en amont, au stade du financement, elle prévoit la mise en place, à compter du 01-01-2009, d'un fonds de péréquation de la santé (Gesundheitsfonds) qui répartit les ressources en fonction des profils de risque des différentes caisses.

26 avril : Présentation de l'Agenda climatique 2020 par le ministre SPD de l'environnement Sigmar Gabriel (économies d'énergie, promotion des énergies renouvelables, réduction de la consommation de carburants et réduction des émissions de gaz à effet de serre).

25 mai-6 juillet : Série de mesures portant réforme de la fiscalité des entreprises avec pour objectif la réduction de la charge fiscale des sociétés anonymes de $39 \%$ à $30 \%$ des bénéfices au 01-01-2008. A partir de 2009, un prélèvement libératoire de $25 \%$ sera appliqué aux revenus du capital. Cette forte réduction du taux d'imposition global est en partie atténuée par un élargissement de l'assiette et des modalités d'amortissement plus restrictives.

24 juillet : Privatisation partielle de la Deutsche Bahn AG. Conflits avec le syndicat des conducteurs de locomotives en vue de l'obtention d'une convention collective spécifique.

25 octobre : Le Bundestag adopte la loi sur le financement de la garde des enfants qui prévoit, grâce à un concours de 4 milliards $€$ du Bund aux Länder, de tripler le nombre de crèches à l'horizon 2013. Le Bundesrat donne son aval le 2512. Par cette initiative, la ministre de la famille, Ulla van der Leyen, met en place le second volet d'une politique familiale active visant à permettre aux femmes de pouvoir concilier activité professionnelle et fonction maternelle.

21 novembre : Franz Müntefering se retire du gouvernement pour raisons privées. II est remplacé aux fonctions de vice-chancelier par Franz W. Steinmeier et à celle de ministre du Travail par Olaf Scholz.

Décembre : Le débat budgétaire permet de dresser un premier bilan de la consolidation financière sous le double effet de la reprise de la croissance en 2006 et de la revalorisation de la TVA en 2007. Alors que le déficit budgétaire était redescendu de $-3,5 \%$ du PIB en 2005 à $-1,6 \%$ en 2006, le budget public global retrouve un solde positif de $0,1 \%$ en 2007 . La dette, quant à elle, a été stabilisée en 2006 et a commencé à décroître en 2007 pour s'établir à 65,7 \% du PIB. 


\section{L'Allemagne à nouveau confrontée à la crise}

2008

$1^{\text {er }}$ janvier : Ouverture totale à la concurrence du marché des services postaux. Instauration le même jour d'un salaire minimum sectoriel fixé par décret du ministère fédéral du Travail ; à la suite de différents recours, cette décision, très vivement débattue entre les partenaires sociaux, fait l'objet d'un examen devant le Tribunal administratif fédéral et d'une saisine du TCF au regard de sa compatibilité avec le principe constitutionnel de l'autonomie contractuelle (Tarifautonomie).

Nouvel abaissement du taux de cotisation chômage de 4,2 \% à 3,3 \% du salaire antérieur, rendu possible par l'amélioration très sensible intervenue sur le marché du travail. Cette dernière justifie au yeux du gouvernement une prolongation de la durée maximale de versement de l'assurance chômage de 18 à 24 mois pour les salariés les plus âgés à compter du 01-03.

4 février : Le TCF adapte les droits fondamentaux à l'ère du numérique. Sur la base de deux droits inscrits dans la Loi fondamentale - l'inviolabilité du secret de correspondance (art. 10) et l'inviolabilité du domicile (art. 13) -, ainsi que de sa jurisprudence sur le droit à l'information et au libre épanouissement de la personnalité, il crée un nouveau droit fondamental : " la garantie de la confidentialité et de l'intégrité des systèmes des technologies de l'information ", surnommé " droit fondamental informatique » (Computergrundrecht). Le recours aux perquisitions en ligne n'est possible que dans certaines conditions, strictement encadrées.

Mars : Le syndicat des conducteurs de locomotives de la Deutsche Bahn obtient sa convention collective catégorielle, ce qui, dans les services publics désormais ouverts à la concurrence, bat en brèche le principe de la convention unitaire de branche.

Mars-avril : Le système bancaire allemand montre des signes de vulnérabilité inquiétants à la suite de la crise financière américaine du subprime. Plusieurs établissements sont menacés d'insolvabilité (Mittelstandsbank IKB, Bayern LB, West $L B$ ), sont rachetés (Dresdner Bank) ou doivent procéder à des dépréciations d'actifs (Deutsche Bank). La crise bancaire s'étend dans le courant de l'été et s'aggrave encore à l'automne avec des menaces de faillite, à l'instar d'Hypo Real Estate. En comparaison, l'économie « réelle » allemande reste portée jusqu'au $3^{\mathrm{e}}$ trimestre par une dynamique industrielle soutenue, nourrie par l'exportation.

Automne : Amorcé pendant l'été, le retournement de conjoncture s'amplifie sous l'effet du déferlement de la crise bancaire en Europe et de la crise de confiance qui s'installe du fait de la raréfaction du crédit. Avec un recul de son PIB de 2,1\% au dernier trimestre, l'Allemagne entre alors brutalement en récession.

13 octobre : Le gouvernement Merkel donne priorité à la prévention du risque systémique avec la création du «Fonds spécial de stabilisation du marché financier » (Sonderfonds Finanzmarktstabilisierung, SoFFin) doté de 480 milliards $€$ permettant d'assurer la garantie des prêts interbancaires, la recapitalisation des établissements et le rachat d'actifs à risques.

Dans un deuxième temps, et dans le contexte du débat européen qui s'engage sur une relance coordonnée, l'Allemagne se montre soucieuse de ne pas compromettre, par des réponses disproportionnées, la consolidation de ses comptes publics.

Avec le «Programme pour garantir l'emploi et dynamiser la croissance " (Konjunkturpaket I) adopté le 5 décembre, elle opte pour une relance sélective fondée sur l'investissement. Celui-ci, d'un encours d'environ 50 milliards $€$, prévoit de soutenir l'accès des entreprises, notamment des PME, au crédit, de promouvoir l'investissement par des mesures fiscales et par l'avancement des programmes publics de modernisation des infrastructures, et accessoirement des mesures fiscales de soutien à la demande des ménages.
Débat sur le salaire minimum

Consolidation de

l'assurance chômage

Droit fondamental numérique

Corporatisme catégoriel à la Deutsche Bahn?

Crise du système bancaire, mais bonne tenue de l'économie réelle

Une gestion graduelle de la crise

Konjunkturpaket I 


\section{9}

Konjunkturpaket II Janvier : Devant la décélération rapide de l'activité constatée en fin d'année dans tous les secteurs de l'industrie, notamment de l'industrie automobile, le gouvernement met en place un second programme de soutien conjoncturel, intitulé « Pacte pour l'emploi et la stabilité en Allemagne », qui porte à nouveau sur 50 milliards $€$ étalés sur 2 ans et financé, comme le précédent par le Bund, les Länder et les communes. II comporte des mesures de soutien à l'investissement (dépenses d'infrastructure) et à l'innovation (programme ZIM pour les PME), de soutien sectoriel (automobile, avec la prime à la casse de $2500 €$ ) et, cette fois, un volet conséquent ( 29 milliards $€$ ) de soutien à la demande des ménages (allègement de l'IR pour les bas salaires, baisse des cotisations maladie et chômage, allongement de la durée du chômage partiel, mesures en faveur des familles). Ce plan a été définitivement adopté le 20 février.

Fonds de soutien aux entreprises

La RFA confrontée à la plus grave récession de son histoire
Mi-mars : A ces mesures vient s'ajouter la mise en place d'un Fonds de soutien aux entreprises (Wirtschaftsfonds Deutschland) doté de 115 milliards $€$ et comportant des aides et garanties publiques à destination des entreprises en butte à la restriction du crédit bancaire.

L'impact budgétaire global de ces mesures de soutien devrait à lui seul se traduire en 2009 par une hausse du déficit de l'ordre de $1 \%$ du PIB. Sous l'effet de la récession, celui-ci devrait atteindre $3,7 \%$ du PIB en 2009 , puis grimper à $5,5 \%$ en 2010, dépassant ainsi nettement son niveau culminant de 2003 (4 \%).

Mai 2009 : L'économie allemande continue de s'enfoncer dans la crise sous l'effet du recul de la demande mondiale provoquée par la crise financière de l'automne. L'Allemagne enregistre un recul de $20 \%$ de ses exportations, une forte montée du chômage partiel (670 000 salariés en mars) et une dégradation désormais sensible du marché de l'emploi. Le chômage refranchit la barre des 3,5 millions de demandeurs d'emploi et devrait atteindre les 4 millions à l'automne. Un certain nombre de grandes entreprises industrielles, au premier rang desquelles le constructeur automobile Opel, sont menacées.

Les prévisions de croissance pour l'année 2009 qui tablaient encore sur une baisse du PIB de $2,5 \%$ à $3 \%$ en janvier sont désormais fortement révisées à la baisse : avec un recul attendu de $6 \%$ de son PIB, la République fédérale d'Allemagne devrait connaître, en cette année de soixantième anniversaire, la plus grave récession économique de son histoire.

$\bullet \bullet$ 